\title{
REVIEW
}

\section{The aortic root: structure, function, and surgical reconstruction}

\author{
M J Underwood, G El Khoury, D Deronck, D Glineur, R Dion
}

Aortic valve insufficiency may be caused by abnormalities of the leaflets, the root, or a combination of both. In some patients, the primary pathology is confined to the aortic root itself, the leaflets remaining anatomically normal. These patients have progressive dilatation of the aortic sinuses and, on occasion, dilatation and distortion of the annulus which results in valvar incompetence. ${ }^{1}$ Most cases are "idiopathic" (annuloaortic ectasia) but it may be associated with a wide spectrum of pathological conditions which include the Marfan syndrome, ${ }^{2}$ aortic dissection and aortitis, ${ }^{34}$ along with rare systemic disorders such as Ehlers-Danlos syndrome.

Aortic root pathology has now been reported as the most common cause of aortic valve incompetence in the United States, an observation which probably reflects the decline of rheumatic valve disease. ${ }^{1}$ Current conventional treatment for patients with significant aortic incompetence caused by a dilated, aneurysmal aortic root is replacement of the ascending aorta using a synthetic graft, replacement of the aortic valve with a mechanical prosthesis (the graft and prosthesis are usually combined as a "composite graft"), and reimplantation of the coronary arteries. In selected cases where there is no suspicion about the future integrity of the sinuses but there is dilatation of the ascending aorta, the valve and ascending aorta may be replaced separately. Despite the success of these operations, they both involve implantation of a prosthetic valve; complications such as thromboembolism, endocarditis, and problems related to the long term anticoagulation required have provided the impetus for the development of a surgical procedure which will preserve the native aortic valve.

In this article we review the structure and function of the aortic root, the pathophysiological changes that may lead to aortic incompetence despite anatomically normal valve leaflets, and the surgical procedure which has been developed as a result of a better understanding of the anatomy and physiology of the aortic root during which the native aortic valve is preserved.

\section{Aortic root: structure}

The aortic root may be defined as the portion of the left ventricular outflow tract which supports the leaflets of the aortic valve, delineated by the sinotubular ridge superiorly and the bases of the valve leaflets inferiorly. ${ }^{6}$ It comprises the sinuses, the aortic valve leaflets, the commissures, and the interleaflet triangles.

The sinuses are expanded portions of the aortic root which are confined proximally by the attachments of the valve leaflets and distally by the sinotubular junction. They are named according to the coronary arteries arising from them - right, left, and non-coronary. The sinotubular junction, delineating the superior aspect of the aortic root, is circular and composed of primarily elastic tissue, and it supports the peripheral attachments of the valve leaflets (fig 1).

The valve leaflets are the portions of the aortic root which separate, haemodynamically, the aorta and the left ventricle. They are inserted into the wall of the root in a semilunar fashion, and the base of the aortic root is defined by the nadirs of attachment of these leaflets. The term "annulus" is frequently used to describe the area of collagenous condensation at the point of leaflet attachment, particularly in surgical literature, but it should be recognised that this term implies a circular structure and as such is inaccurate. ${ }^{7}$ The posterior aspect of the aortic root (mainly non-coronary leaflet) is supported by fibrous tissue for approximately $55 \%$ of its circumference (membranous part of the septum to the left fibrous trigone), ${ }^{7}$ while the remainder is supported by ventricular muscle, an important distinction when performing a

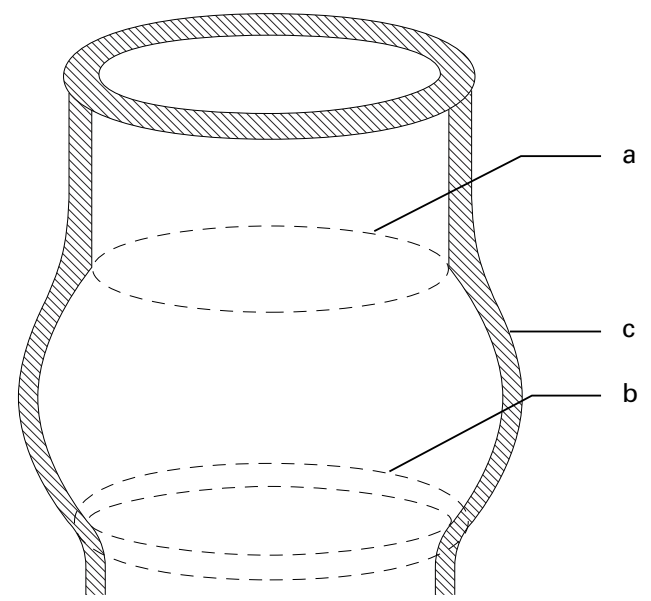

Figure 1 Diagrammatical representation of the aortic root: (a) sinotubular junction; (b) basal ring (surgical annulus); (c) the sinuses of Valsalva. 


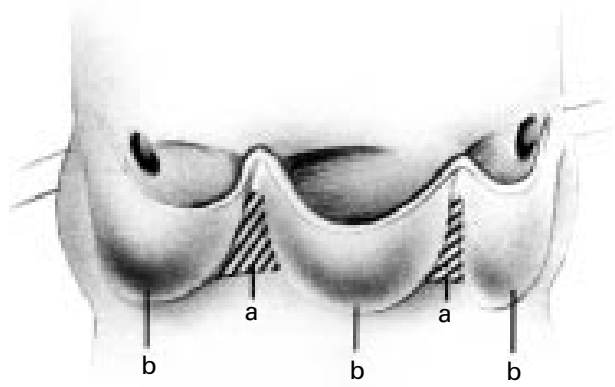

Figure 2 Diagrammatic representation of the aortic root opened longitudinally through the left coronary sinus, demonstrating the interleaflet triangles (a) and the valve leaflets (b).

surgical annuloplasty as will be discussed later. At the midpoint of the free edge of each aortic valve leaflet is a fibrous nodule, on either side of which is a thin crescent shaped portion of the leaflet called the "lannula" which are occasionally fenestrated near the commissures. The apices of attachment of the valve leaflets are at the level of the sinotubular ridge.

The commissures are defined as "those structures where the adjacent attachments of the leaflets run parallel for a short distance". Beneath the apices formed by these lines are areas bounded by the semilunar attachments of the valve leaflets which have recently been well described and called the "interleaflet triangles" (fig 2). ${ }^{6}$

The geometrical relations of the aortic root have been shown to be consistent over a wide range of sizes, an important point when considering surgical reconstruction. The diameter of the sinotubular junction is $10-15 \%$ smaller than the diameter of the annulus. ${ }^{8}$ The upper part of the valve commissures are attached just below the sinotubular junction and the diameter of the aortic root at this level approximates to the diameter of the annulus. The non-coronary sinus is the largest of the three sinuses. The length of the base of the leaflets is approximately 1.5 times longer than the length of its free margin and the height of the leaflets ranges from $12-18 \mathrm{~mm} .{ }^{8}$ As with the sinuses, the non-coronary leaflet is slightly larger than the other two.

The anatomy of the aortic root has been well described, and its constant dimensions defined by both surgeons and morphologists. Of particular interest when considering aortic remodelling is the fact that as a result of these studies an equation has been derived which allows the calculation of the most appropriate root diameter for an individual patient, at the level of the sinuses, based upon leaflet dimensions. ${ }^{89}$ This allows the correct size of graft to be chosen and tailored during reconstructive surgery; its use will be explained in more detail in the surgical technique section of this paper. This anatomical knowledge has also focused interest on the functional complexity of the aortic root.

\section{Aortic root: function}

The function of the aortic valve has often been thought to be entirely passive-when the pressure generated by ventricular systole exceeds that in the ascending aorta the valve leaflets open, and when left ventricular pressure decreases to less than aortic pressure they close. Aortic valve function is, however, much more complicated and the aortic root complex acts as an individual haemodynamic system. ${ }^{10}{ }^{11}$ The upper portion of the aortic root is exposed to aortic pressure changes and therefore behaves as the rest of the vessel in that it expands during systole allowing the leaflets to retract and open. The proximal part of the root complex, however, is exposed to ventricular pressure changes and it will expand as the ventricle fills and contracts during peak systole which decreases the distance the valve leaflets have to travel to coapt. ${ }^{12}$ However, it is the sinuses which seem to serve a most important physiological role in aortic valve function, and their importance has been increasingly recognised by surgeons contemplating reconstruction of the aortic root.

The sinuses play two very important roles. They provide space behind the open aortic leaflets so that the leaflets do not occlude the coronary artery orifices. Secondly, this space favours the development of eddy currents behind the leaflets when they are open. These hold the leaflets away from the aortic wall in a position where they will be promptly caught and closed by blood flow at the end of systole. Scientific study has further clarified the relation between the sinuses and valve function.

Early in systole the leaflets move towards the sinuses and vortices form between the leaflet and the sinus wall. Blood flow enters at the level of the ridge on the sinotubular junction, moves along the wall, and flows back into the main stream. These currents prevent the leaflets striking the aortic wall when the valve opens and are also important in promoting valve closure. After peak systole the currents force the leaflets to move back away from the aortic wall so that they almost totally coapt before the end of systole. ${ }^{13}$ Although it is considered that the formation of these currents is more a function of the sinus ridge than the shape of the sinus, sinus shape is important when considering valve function, the curvature of the sinus probably being important in determining the distribution of stress on the valve leaflets. The shape of the sinus in diastole is spherical and the shape of the leaflets cylindrical. The stress on the leaflets in diastole is almost four times that in the sinuses, and this would result in the sinus walls being drawn inwards in diastole if the stress were not shared by the sinuses. Instead the sinus walls move outward decreasing the stress and wear on the aortic leaflets. ${ }^{14}$ Hence the sinuses can be seen as the basic structural and functional unit of the valve, although recently added importance has been given to the function of the interleaflet triangles, ${ }^{6}$ which are said to allow the sinuses to act independently and are therefore crucial to proper valve function. 


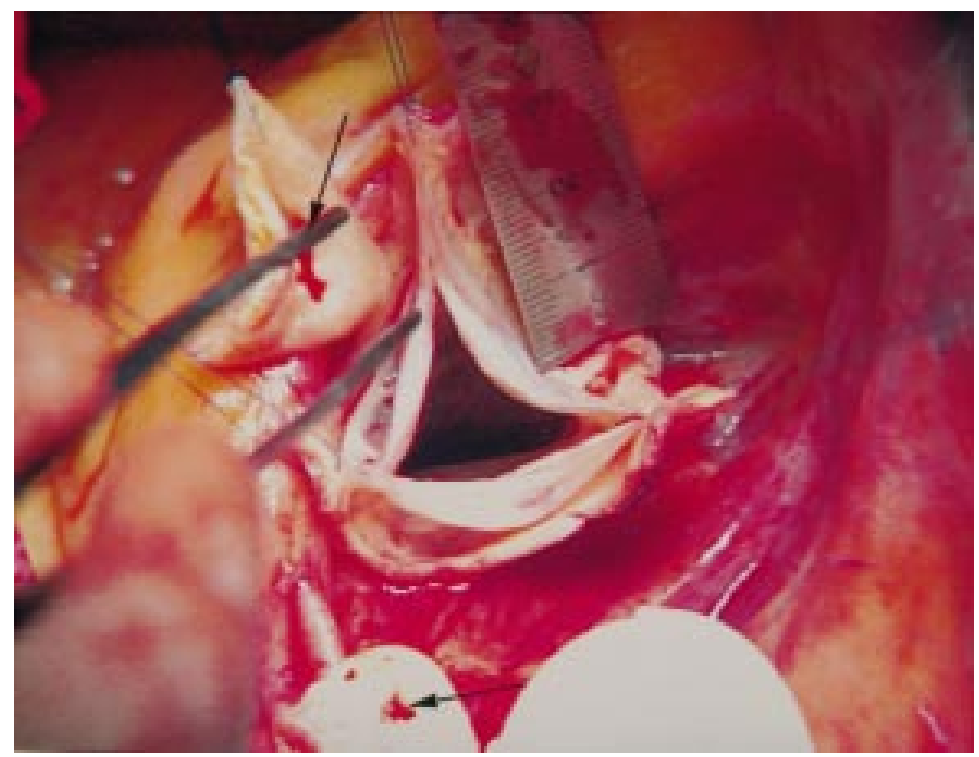

Figure 3 Operative picture: measurement of the height of the commissures.

With this information we can see how any of the previously listed pathological conditions of the aortic root may result in valve incompetence. Valve insufficiency appears because dilatation of the root and sinotubular junction will displace the valve commissures outwards so the leaflet edges cannot coapt in diastole. Disease of the sinuses may also effect competence because of the disruption of the complex role it plays in valve function which we have described. These mechanisms have been recognised for more that one and a half centuries, ${ }^{15} 16$ but it is only in recent times that new surgical techniques have been introduced to preserve the aortic valve at operation, based on a sound understanding of aortic root structure and function as outlined above.

\section{Surgical technique: remodelling of the} aortic root

Patients who have aortic root pathology and normal aortic valve leaflets are suitable for a

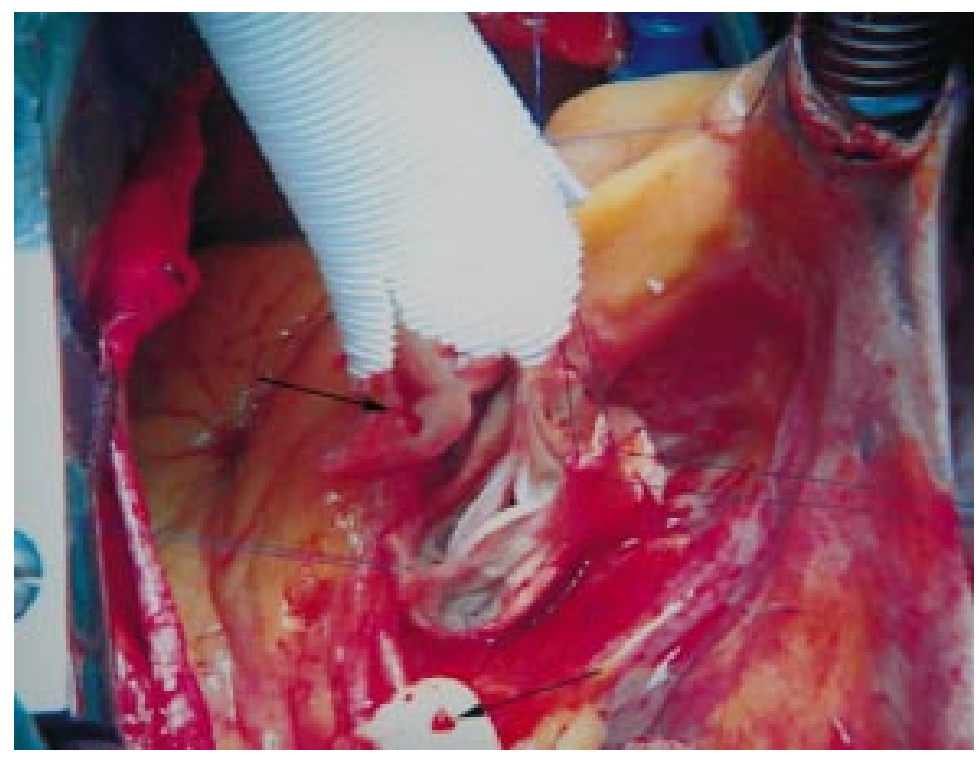

Figure 4 Commencement of graft placement: suturing is commenced at the level of the commissures. remodelling procedure. Along with preoperative echocardiographic and angiographic assessment of the aortic root, it is imperative that perioperative transoesophageal echocardiography (TOE) is used in these patients. This will provide the surgeon with important information regarding the morphology of the aortic root and also the dimensions of the aortic annulus and severity of any aortic regurgitation. If the aortic annulus is also dilated $(>28 \mathrm{~mm}$ ) on echocardiography and during perioperative measurement, the remodelling procedure can be combined with a surgical annuloplasty. TOE must also be used following the procedure to ensure that there is no residual aortic regurgitation.

At surgery, the dilated sinuses and diseased ascending aorta are excised so that only $4-5 \mathrm{~mm}$ of aortic wall is left attached to the annulus. The coronary ostia are also mobilised and prepared for reimplantation. The diameter of the aortic annulus is then measured using metric sizers. If it is not dilated, then based on the previously described aortic root relations, a Dacron graft approximately $10 \%$ smaller than the size of the annulus is chosen to remodel the sinuses. ${ }^{17}$ If the annulus is dilated and an annuloplasty is to be performed, then the leaflet dimensions are measured as described below.

Three equidistant marks are then made on the graft. Other authors suggest the graft should then be incised along these marks for a length of approximately two thirds of the graft diameter. ${ }^{17}$ To estimate more accurately the length of these incisions on the graft, however, we find it easier to suspend the aortic valve commissures with prolene sutures and measure their height (fig 3). The length of the incisions in the graft is made slightly larger than this measurement so scalloping of the graft reproduces the crescent shape of the root and creates "pseudosinuses" in the supravalvar region. ${ }^{18}$ The aortic commissures are then sutured to the appropriate position on the graft (fig 4) and the intervening Dacron sutured to the remnants of the aortic sinuses. Since the perimeter of each scalloped area of the graft is longer than the scalloped aortic annulus it is important that sutures are placed further along the graft than the annulus to create "neo-sinuses"; to achieve this suturing is commenced at the level of the commissures. The coronary arteries are then reimplanted and the graft anastomosed to the distal aorta in conventional fashion. Normal sinuses need not be replaced and sometimes only one sinus is diseased and needs replacing. The most commonly affected seems to be the non-coronary sinus. Figure 5 shows the completed reconstruction and demonstrates the appearance of the neo-sinuses.

If the annulus itself is seen to be dilated, an annuloplasty can be added to the procedure. The dilatation is usually confined to the fibrous portion of the left ventricular outflow tract (the fibrous support of the aortic root described previously) and this is the area reduced with an annuloplasty. This is performed by placing sutures from the inside to the outside of the aortic root in a horizontal plane below the level of the valve leaflets (largely the non-coronary 


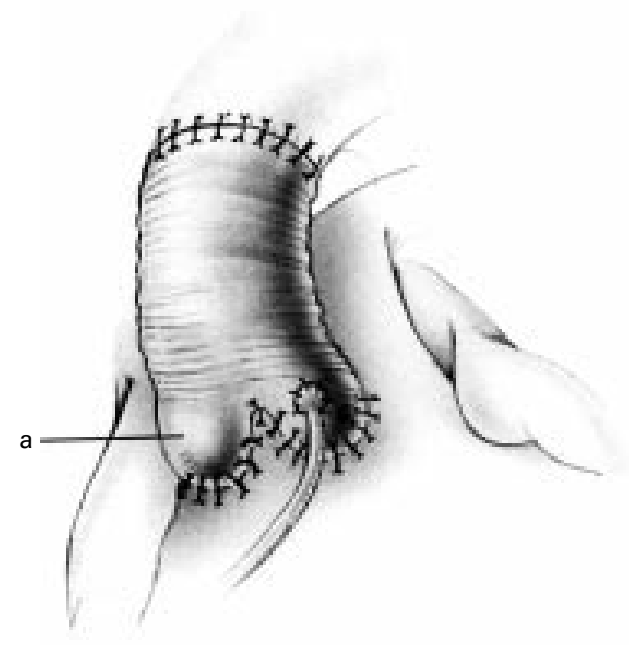

Figure 5 Completed remodelling. The slight bulging of the neo-sinuses is visible (a).

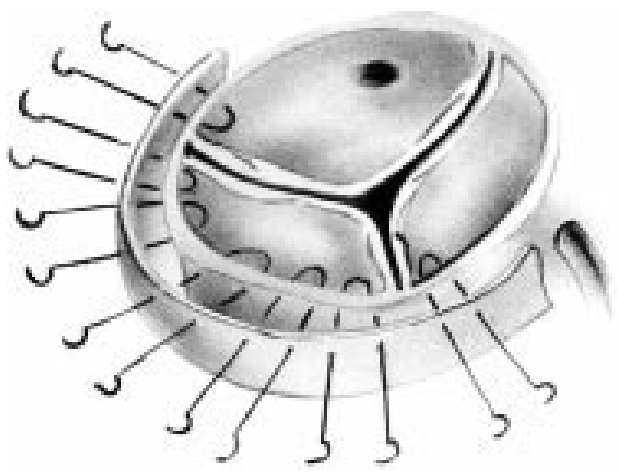

Figure 6 Technique of aortic annuloplasty which may be combined with the remodelling procedure.

leaflet), all along the fibrous portion of the outflow tract, and these sutures are reinforced with a strip of Teflon felt (fig 6). Occasionally, performing an annuloplasty may distort the geometry of the root but this can be compensated for by performing a commissuroplasty at the commissure between the right and left coronary leaflets. To determine the most appropriate diameter graft for a patient with annular dilatation, the length of the free edges of the aortic valve leaflets are measured and averaged. A graft $10 \%$ smaller than these combined lengths is then chosen. ${ }^{9}{ }^{18}$ The remainder of the operation is then performed as in the remodelling procedure already described.

\section{Aortic remodelling: reported clinical results}

Data are available from a few surgical groups who have pioneered this procedure. The largest reported series comes from Yacoub and colleagues who have performed the procedure on 158 patients since $1979 .{ }^{19}$ They report an early (30 day) mortality of $4.6 \%$. Their follow up ranged from 30-6396 days (mean 2025 days) and the actuarial survival at 5, 10, and 15 years was $91.2 \%, 82.0 \%$, and $60.0 \%$, respectively, for patients undergoing elective surgery.
Importantly the probability of freedom from reoperation at 1 and 10 years was $98.8 \%$ and $89.0 \%$, respectively, and these figures were comparable in both Marfan and non-Marfan cases. No patient required anticoagulation and there were no reported instances of endocarditis or thromboembolism. Follow up echocardiography has shown trivial or no aortic regurgitation in $63.6 \%$ of patients, and mild to moderate regurgitation in $33.3 \%$. Echocardiography also showed that the reductions in left ventricular end systolic and end diastolic dimensions were maintained throughout the follow up period.

Following their original reports ${ }^{20}$ David and colleagues have published an update of a series of 126 cases who had aortic valve sparing operations, including 60 patients who had a remodelling procedure which included excision of one or more sinuses. ${ }^{21}$ Their results suggest long term durability with equal results in patients with the Marfan syndrome.

After these original reports, a series of 10 patients was reported by Cochran and colleagues in which they adopted a slight technical modification of the procedure to enhance the creation of neo-sinuses. ${ }^{18}$ They reported no operative mortality and follow up averaged 13.3 months. All patients are in New York Heart Association (NYHA) functional class I or II . Five patients have no residual regurgitation on postoperative echocardiogram, four have a "trace", and one patient has grade I. Repeat echocardiograms at six months showed no progression of regurgitation in any patient and interestingly confirmed the presence of the constructed pseudosinuses. ${ }^{18}$

\section{Discussion}

The mechanism of aortic regurgitation in patients who have dilatation of the sinotubular junction but normal aortic valve leaflets has been appreciated for many years. ${ }^{15}$ The current conventional treatment for these patients consists of aortic root replacement with a composite graft incorporating a prosthetic valve or separate replacement of the ascending aorta and valve. These are successful procedures and nearly all contemporary series report a 30 day mortality of less than $5 \%$ for elective operations. ${ }^{22}$ However, these patients may be young (mean age 46.6 years in the Yacoub series $^{19}$ ) and implantation of a mechanical valve means they require life long anticoagulation and are at risk of the many potential complications which relate to these prostheses, such as thromboembolism and endocarditis. The operative technique of remodelling the aortic root was introduced by Yacoub and David based on a better understanding of the structure and function of the aortic root, and in particular the contribution of the sinuses of Valsalva to valve function. This operation has a major theoretical advantage over conventional root replacement in that the native aortic valve is preserved, thus avoiding the implantation of a mechanical prosthesis. At present, however, several questions remain unanswered.

The data available show that overall this procedure does not have a significantly higher 
mortality than root replacement and the postoperative complication rates are reassuringly low. In our institution we have performed 25 remodelling procedures since July 1995 . Six patients had the Marfan syndrome and six patients underwent surgery for chronic type A aortic dissection. There have been no perioperative deaths and one early death $(<30$ days postoperatively) had a non-cardiac cause (pulmonary aspiration). The surviving patients have been followed up for a mean of 13.5 months. All patients are in NYHA functional class I or II and serial echocardiography has not shown progression of aortic regurgitation. No patient is taking anticoagulants and there have been no cases of endocarditis or episodes of thromboembolism. These results are comparable with the other published series.

The technique is successful in restoring valve competence as demonstrated by the perioperative echocardiogram findings, and postoperative follow up has shown that these findings seem to remain stable although the follow up period is at present short in our series. In the longer term, however, concerns still exist with respect to the potential for late deterioration in valve function and continued follow up is required. ${ }^{23}$ In the remodelling operation, although attention is given to creating neosinuses, particularly using the technique described by Verrier and colleagues, the operation is carried out using a non-compliant tube (Dacron) which in the long term may have a detrimental effect on the function of the aortic valve because of leaflet damage. Only the follow up of increasing numbers of patients for longer periods of time can truly answer the question regarding the durability of the remodelling procedure. It may also be that, in the future, prosthetic materials can be developed which mimic more closely the dynamic movements of the aortic root and help maintain the important equilibrium required for valve function.

Another concern is the use of this technique in patients with the Marfan syndrome because of the potential for ongoing annular dilatation and valve degeneration. Recent histological studies have shown a structural deterioration of the aortic leaflets in patients with Marfan's and a diminished fibrillin count. ${ }^{22}$ Fibrillin is a glycoprotein which helps maintain the structural integrity of connective tissue including the aortic wall and valve leaflets. This seems to suggest that a cautious approach should be adopted when considering valve sparing operations in this group of patients. More concrete contraindications agreed by the proponents of this technique include bicuspid aortic valves or deformed valve leaflets. Endocarditis has been reported as being the most common late complication of conventional root replacement but so far no author has reported a case of endocarditis following aortic remodelling. Thromboembolism has also been reported after root replacement in the setting of inadequate anticoagulation, a problem eliminated by the valve sparing procedure.

Surgical reconstruction of the aortic root for patients with aortic dilatation and associated valve incompetence has been adopted as a surgical technique in preference to root replacement by a few active proponents of this procedure. Early results are encouraging and the problems of endocarditis and thromboembolism seen in patients following conventional surgery seem to have been virtually eliminated. Only long term follow up will determine the durability of this technique and clarify whether the purported theoretical advantages are translated into clinical reality.

1 Olson LJ, Subramanian R, Edwards WD. Surgical pathology of pure aortic insufficiency: a study of 225 cases. Mayo Clin $\operatorname{Proc} 1984 \cdot 59 \cdot 835-41$

2 Roberts WC, Honig HS. The spectrum of cardiovascular disease in the Marfan syndrome: a clinico-morphologic study of 18 necroscopy patients and comparison to 151 previously reported necroscopy patients. Am Heart f 1982; 104:115-35.

3 Larson EW, Edwards WD. Risk factors for aortic dissection: necroscopy study of 161 cases. Am $\mathcal{F}$ Cardiol 1984;53: 849-55.

4 How J, Strachan RW. Aortic regurgitation as a manifestation of giant cell arteritis. Br Heart 7 1978;40:1052-4.

5 Leier CV, Call TD, Fulkerson PK, et al. The spectrum of cardiac defects in the Ehlers-Danlos syndrome, types I and III. Ann Intern Med 1980;92:171-8.

6 Sutton JP, Ho SY, Anderson RH. The forgotten interleaflet Sutton JP, Ho SY, Anderson RH. The forgotten interleaflet
triangles: a review of the surgical anatomy of the aortic triangles: a review of the surgical anat
valve. Ann Thorac Surg 1995;59:419-27.

7 Anderson RH, Devine WA, Ho SY, et al. The myth of the aortic annulus: the anatomy of the subaortic outflow tract. Ann Thorac Surg 1991;52:640-6.

8 Kunzelman KS, Grande KJ, David TE, et al. Aortic root and valve relationships. Impact on surgical repair. $\mathcal{F}$ Thorac Cardiovasc Surg 1994;107:162-70.

9 David TE. Aortic valve repair in patients with Marfan's syndrome and ascending aorta aneurysms due to degenerative disease. F Card Surg 1994;9(suppl):182-7.

10 David TE. An anatomic and physiologic approach to acquired heart disease. Eur $\mathcal{F}$ Cardiothorac Surg 1995;9: 175-80.

11 Brewer RJ, Deck JD, Capati B, et al. The dynamic aortic root. $\mathcal{F}$ Thorac Cardiovasc Surg 1976;72:413-7.

12 Gould PL, Cataloglu A, Dhatt C, et al. Stress analysis of the human aortic valve. Comput Sci 1973;3:377-86.

13 Thrubrikar M, Nolan SP, Bosher LP, et al. The cyclical changes and the structure of the base of the aortic valve. Am Heart F 1980;99:217-24

14 Thubrikar MJ, Nolan SP, Aouad J, et al. Stress sharing between the sinus and leaflets of the canine aortic valve. Ann Thorac Surg 1986;42:434-40

15 Bellhouse BJ, Bellhouse F, Abbot JA, et al. Mechanism of valvular incompetence in aortic sinus dilatation. Cardiovasc Res 1973;7:490-4.

16 Corrigan DJ. Permanent patency of the mouth of the aorta. Edinburgh Med Surg 1832;37:37-111.

17 David TE, Feindel CM, Bos J. Repair of the aortic valve in patients with aortic insufficiency and aortic root aneurysm. patients with aortic insufficiency and aortic roor
F Thorac Cardiovasc Surg 1995;109:345-52.

18 Cochran RP, Kunzelman KS, Eddy AC, et al. Modified conduit preparation creates a pseudosinus in an aortic valve-sparing procedure for aneurysm of the ascending aorta. $\mathcal{F}$ Thorac Cardiovasc Surg 1995;109:1049-58.

19 Yacoub M, Gehle P, Chandrasekaran V, et al. Late results of a valve preserving operations in patients with aneurysms of the ascending aorta and root. $\mathcal{F}$ Thorac Cardiovasc Surg 1998;115:1080-90.

20 David TE, Feindel CM. An aortic valve-sparing operation for patients with aortic incompetence and aneurysm of the ascending aorta. F Thorac Cardiovasc Surg 1992;103:61722.

21 David TE, Armstrong S, Ivanov J, Webb GD. Aortic valve sparing operations: an update. Ann Thorac Surg 1999;67: 1840-2.

22 Gott VL, Laschinger JC, Cameron DE, et al. The Marfan syndrome and the cardiovascular surgeon. Eur $\mathcal{F}$ Cardiovasc Surg 1996;10:149-58.

23 Luciana GB, Casali G, Tomezzoli A, et al. Recurrence of aortic insufficiency after aortic root remodelling with valve preservation. Ann Thorac Surg 1999;67:1849-52. 